\title{
Interrogating RNA-small molecule interactions with structure probing and Al augmented-molecular simulations
}

\author{
Yihang Wang, 1, a) Shaifaly Parmar, ,2, a) John S. Schneekloth, Jr, 2, b) and Pratyush Tiwary, c) \\ ${ }^{1)}$ Biophysics Program and Institute for Physical Science and Technology, University of Maryland, College Park, \\ $M D$ 20742, USA. \\ ${ }^{2)}$ Chemical Biology Laboratory, Center for Cancer Research, National Cancer Institute, Frederick, MD 21702, \\ USA. \\ ${ }^{3)}$ Department of Chemistry and Biochemistry and Institute for Physical Science and Technology, \\ University of Maryland, College Park 20742, USA.
}

(Dated: 28 September 2021)

While there is increasing interest in the study of RNA as a therapeutic target, efforts to understand RNAligand recognition at the molecular level lag far behind our understanding of protein-ligand recognition. This problem is complicated due to the more than ten orders of magnitude in timescales involved in RNA dynamics and ligand binding events, making it not straightforward to design experiments or simulations. Here we make use of artificial intelligence (AI)-augmented molecular dynamics simulations to directly observe ligand dissociation for cognate and synthetic ligands from a riboswitch system. The site-specific flexibility profiles from our simulations are in excellent agreement with in vitro measurements of flexibility using Selective 2' Hydroxyl Acylation analyzed by Primer Extension and Mutational Profiling (SHAPE-MaP). Our simulations reproduce known binding affinity profiles for the cognate and synthetic ligands, and pinpoint how both ligands make use of different aspects of riboswitch flexibility. On the basis of our dissociation trajectories, we also make and validate predictions of pairs of mutations for both the ligand systems that would show differing binding affinities. These mutations are distal to the binding site and could not have been predicted solely on the basis of structure. The methodology demonstrated here shows how molecular dynamics simulations with all-atom force-fields have now come of age in making predictions that complement existing experimental techniques and illuminate aspects of systems otherwise not trivial to understand.

\section{INTRODUCTION}

RNA regulates diverse cellular processes, far beyond coding for protein sequence or facilitating protein biogenesis. ${ }^{1}$ In this role, RNA can interact with protein $^{2}$ or small molecule factors ${ }^{3,4}$ to directly or indirectly impact gene expression and cellular homeostasis. For example, riboswitches are sequences found primarily in bacterial mRNAs that contain aptamers for small molecules and regulate transcription or translation. ${ }^{5}$ In humans, mutations in noncoding RNAs can directly impact neurodegenerative diseases and cancer. ${ }^{6,7}$ The regulatory role of bacterial riboswitches, coupled with the broader link between RNA and human disease has also led to interest in RNA as a therapeutic target for small molecules. ${ }^{8-10}$ However, in comparison to proteins, methods to computationally and experimentally interrogate RNA structure are relatively less mature. Thus, more robust tools are needed to better understand RNA structure, dynamics, and recognition of small molecules. In spite of its clear relevance to fundamental science and drug discovery efforts, the highly dynamic nature of RNA makes it difficult to study due to the diverse conformational ensembles it adopts. ${ }^{11,12}$ Luckily, riboswitches rep-

\footnotetext{
a) These two authors contributed equally.

b) Electronic mail: schneeklothjs@mail.nih.gov; Co-corresponding author.

c)Electronic mail: ptiwary@umd.edu; Co-corresponding author.
}

resent a valuable model for RNA-small molecule recognition. When ligands bind to riboswitch aptamers, the RNA undergoes a conformational change that modulates gene expression..$^{13,14}$ The cognate ligand for a riboswitch is typically associated with its upstream gene, often part of the ligand's biosynthetic pathway. ${ }^{14,15}$ This feedback mechanism, paired with well-established structural analyses, make riboswitches excellent models for RNA-ligand binding since the target ligand is known and often highly specific. ${ }^{14}$

Although we can use riboswitches to model RNAsmall molecule interactions, visualizing riboswitch-ligand dissociation pathways with high spatiotemporal resolution remains a challenge for in vitro and in silico experiments. Such a femtosecond and all-atom resolution is hard to directly achieve in in vitro experiments, while the associated timescales are several orders of magnitude too slow for molecular dynamics (MD) simulations performed even on the most powerful supercomputers. Previously, Ref. 16 used G $\bar{o}$-model simulations of $\mathrm{PreQ}_{1}$ riboswitches, specifically transcriptional Bacillus subtilis $(B s)$ and translational Thermoanaerobacter tengcongensis (Tt) aptamers. Their work suggests that in respective cases the ligand binds at late and early stages of riboswitch folding, indicating that perhaps these riboswitches respectively fold via mechanisms of conformational selection and induced fit. Since Ref. 16, RNA force-fields have become more detailed and accurate, but the timescales involved in RNA-small molecular dissociation continue to stay beyond reach. ${ }^{17}$ 

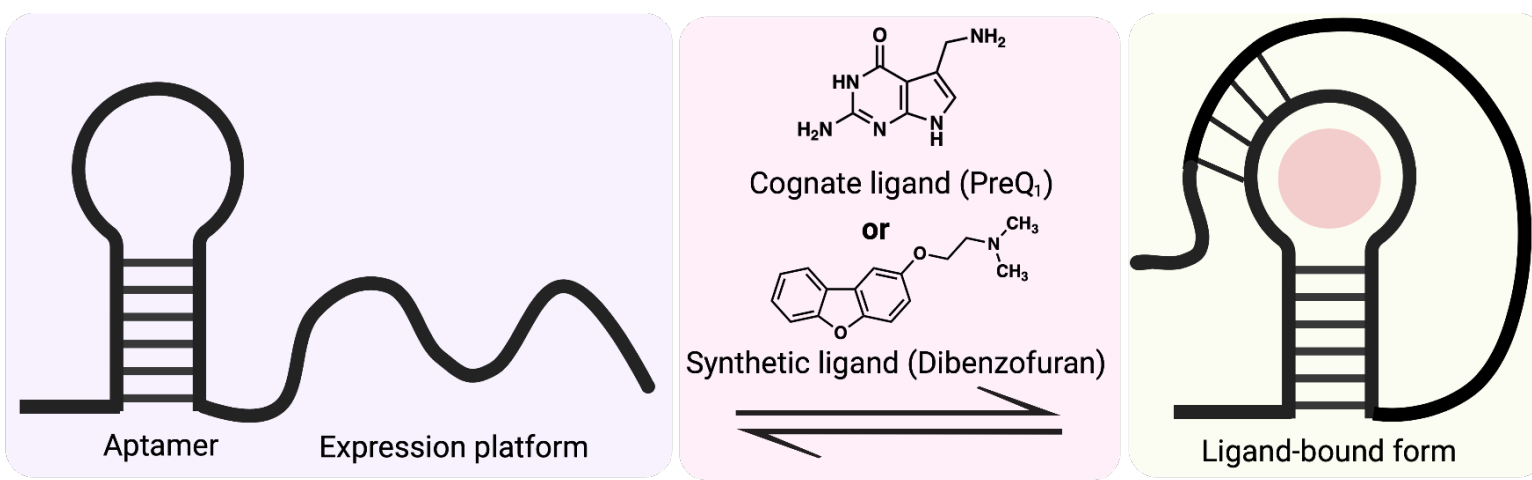

FIG. 1: Conformational change of the $P_{r e Q}$ riboswitch and chemical structures of $P_{r e} Q_{1}$ and synthetic dibenzofuran ligands.

In order to better understand the ligand-riboswitch dissociation process, in this work we employ a combination of (i) enhanced sampling methods combining statistical physics with artificial intelligence $(\mathrm{AI})^{18-21}$ and (ii) experimental techniques to measure RNA flexibility and ligand binding. ${ }^{22-24}$ The computational sampling techniques allow us to study the dissociation process in an accelerated but controlled manner, while the Seletive 2' Hydroxyl Acylation analyzed by Primer Extension and Mutational Profiling (SHAPE-MaP), microscale thermophoresis (MST) and fluorescence intensity assay (FIA) provide a rigorous validation of the computational findings. Specifically, we study the Tt$\mathrm{PreQ}_{1}$ riboswitch aptamer interacting with its cognate ligand $\mathrm{PreQ}_{1}$ (7-aminomethyl-7-deazaguanine) and a synthetic ligand (2-[(dibenzo[b,d]furan-2-yl)oxy]-N,Ndimethylethan-1-amine). ${ }^{25}$ First, we perform $2 \mu$ s long unbiased MD simulations of the ligand-bound and ligandfree systems to quantify the flexibility of individual nucleotides. We find these to be in excellent agreement with SHAPE-MaP measurements. To simulate the dissociation process, which is too slow for MD simulations, ${ }^{17}$ a key challenge in most enhanced sampling methods is the need for a priori estimate of the dominant dissociation mechanisms as captured through the reaction coordinate (RC). However, it is very challenging to estimate such an $\mathrm{RC}$ without already having simulated the dissociation process, especially for flexible biomolecules like RNA. ${ }^{26}$ Here, we use our AI-based sampling method RAVE to directly observe the dissociation of both of these ligands from the wild-type (WT) Tt-PreQ 1 riboswitch. RAVE automates the learning of RCs through a scheme that iterates between $\mathrm{MD}$ to generate data and deep learning to construct an approximate RC from this data. The MD-deep learning iteration continues until the RC estimate converges. The RC itself is learned as the most informative low-dimensional representation expressed as a past-future information bottleneck. ${ }^{27}$

By efficiently sampling multiple independent riboswitch-ligand dissociation events for both cognate and synthetic ligands, we are able to reproduce binding affinity and flexibility measurements from MST, FIA and SHAPE-MaP experiments. Furthermore, we are able to pin-point which nucleotides play the most critical roles in dissociation. These are found to be the nucleotides U22 and A32 for the cognate and synthetic ligands respectively. On the basis of these we design and perform a set of in vitro mutagenesis experiments, which validate our mutation predictions. We note that these predicted mutations are unlikely to have been found simply from a structural perspective, as they are distal from the binding site and do not directly interact with either of the ligands. Our findings thus reveal the complex nature of interaction between RNA and small molecules, and might help guide the design of RNA-binding small molecules in other systems in the future. Thus, this methodology will be broadly useful in understanding increasingly complex, biologically important RNA-small molecule interactions.

\section{RESULTS}

\section{A. Ligand binding induces flexibility change in riboswitch aptamer}

Our first test is to compare the nucleotide-specific flexibility from all-atom simulations to those from SHAPEMaP measurements. We perform $2 \mu$ s unbiased MD simulations (4 independent sets of 500 ns each for both ligands) each for $\mathrm{PreQ}_{1}$ riboswitch bound with the cognate and synthetic ligand respectively. See Supplementary materials (SM) for details of simulation set-up. While these simulations are not long enough to observe ligand dissociation, they can still give a robust measure of how the flexibility varies between the two different ligand-bound systems and provide nucleotide-level insight into how specific ligand binding induces different conformation changes in the riboswitch. Specifically, we compare the fluctuations of the distances between consecutive $\mathrm{C} 2$ atoms obtained from these simulations with SHAPE-MaP measurements (see SM for details of 
SHAPE-Map). We performed SHAPE-MaP analyses using a PreQ $Q_{1}$ riboswitch construct consisting of the entire riboswitch (both the aptamer domain and expression platform). Probing was performed using a recently reported SHAPE reagent, 2A3, that provides improved mutagenesis rates during reverse transcription. ${ }^{28} \mathrm{We}$ analyzed data in the absence of any ligand, and in the presence of either cognate ligand $\left(\mathrm{PreQ}_{1}\right)$ or synthetic ligand. Our results were in good agreement with previous studies reporting SHAPE-MaP on $\mathrm{PreQ}_{1}$ riboswitches using different SHAPE reagents and a slightly shorter construct. ${ }^{29}$ As discussed previously, ${ }^{30}$ base pairing interactions are the most important determinant of RNA structural constraints, and thus base-base distance fluctuations are a crucial metric of RNA backbone flexibility. Indeed, as shown in Fig. 2, we obtain excellent agreement between $\mathrm{C} 2-\mathrm{C} 2$ distances from unbiased $\mathrm{MD}$ and experimentally measured SHAPE flexibilities for all 3 systems: ligand-free $\mathrm{PreQ}_{1}$ aptamer, cognate-ligandbound $\mathrm{PreQ}_{1}$ aptamer and synthetic-ligand-bound aptamer. In addition to the strong agreement between MD and SHAPE, Fig. 2 also shows that, for all three systems, nucleotides in the ranges A13-C15, U21-A24, and A32-G33 are more flexible than other regions of the riboswitch. ${ }^{29}$ The agreement between MD and SHAPE approaches provides solid validation that our simulations using classical force-fields ${ }^{31}$ accurately model the behavior of complex RNAs.

\section{B. Reaction coordinates and free energy profile}

Encouraged by the agreement between flexibility profiles from unbiased MD and SHAPE, we next aim to calculate the free energy profiles and reaction coordinates for ligand dissociation from $\mathrm{PreQ}_{1}$. As these timescales are far beyond unbiased MD, here we use our recent AIaugmented MD method RAVE which learns the dominant slow degrees of freedom on-the-fly by iterating between rounds of sampling and AI. See SM for further details of the protocol. After four rounds of such iterations, we obtain converged RCs for both systems, expressed as a linear combination of different riboswitch-ligand heavy atom contacts. Fig. 3 shows different contacts and their weights as obtained from RAVE simulations. It is interesting to note that the $\mathrm{RC}$ for the cognate ligand has many more components than the RC for synthetic ligand, highlighting differences between the molecules. Most of these contacts in the $\mathrm{RC}$ are present also in the crystal structure (PDB 6E1W, 6E1U), except carbon 5-U2, carbon $5-\mathrm{A} 23$ for the cognate ligand and carbon 1-A23 for the synthetic ligand (ligand atom numbering corresponds to numbering in PDB files). We then used this optimized $\mathrm{RC}$ and ran 10 independent biased MD simulations each using well-tempered metadynamics ${ }^{21}$ to sample the free energy profile of the systems. In Fig. 4 we provide the free energy profiles as a function of the ligand-riboswitch coordination number for both systems. Note that for

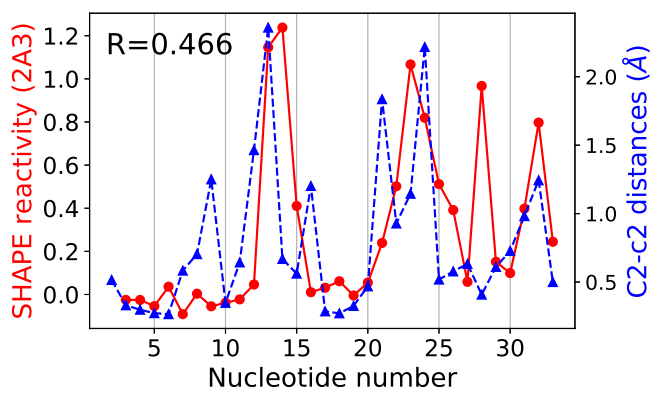

(a)

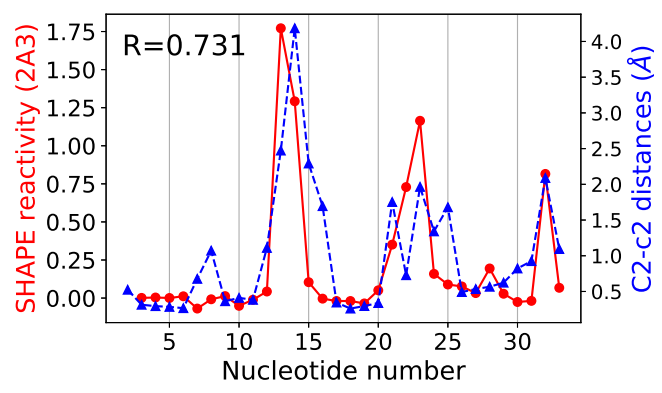

(b)

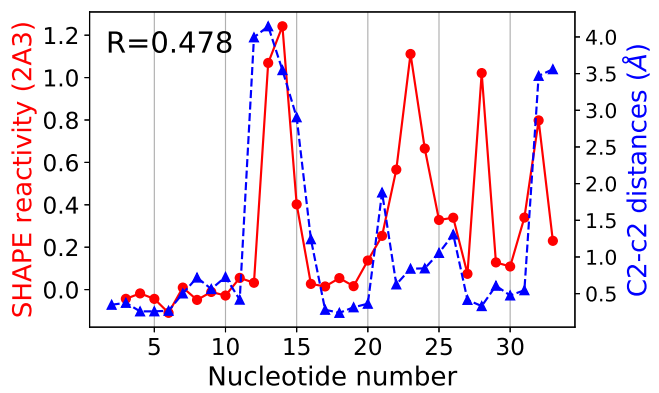

(c)

FIG. 2: SHAPE reactivities for the $T t \mathrm{PreQ}_{1}$ riboswitch aptamer obtained using the $2 \mathrm{~A} 3$ reagent (red circles joined by solid lines) are compared with the fluctuations of $\mathrm{C} 2-\mathrm{C} 2$ distances (blue triangles joined by dashed lines) for the $\mathrm{PreQ}_{1}$ riboswitch (a) in the absence of ligand, (b) bound to cognate ligand, and (c) bound to synthetic ligand. Pearson correlation coefficients $R$ are shown in upper left corners.

both systems there were 2 predominant pathways as indicated through arrows in Fig. 5. A key difference between the two paths is that in the first path, the ligand unbinds through the space in between stem 2 and the backbone connecting stem 1 and loop 1 (Fig. 5, blue arrows). In the second path, the ligand unbinds through the space in between loop 2 and stem 2 (Fig. 5, red arrows). In order to compare the binding free energies of the two systems, we combine the information from both the pathways by plotting the free energy as a function of the ligand-riboswitch shared coordination number $C$. During the dissociation process, the coordination num- 


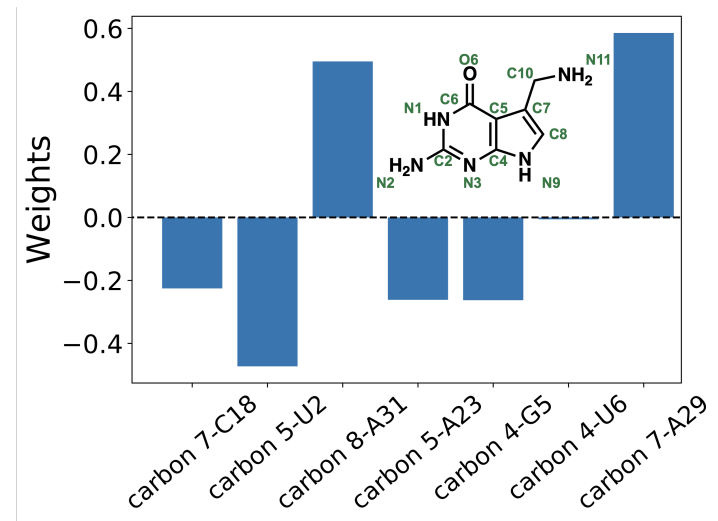

(a)

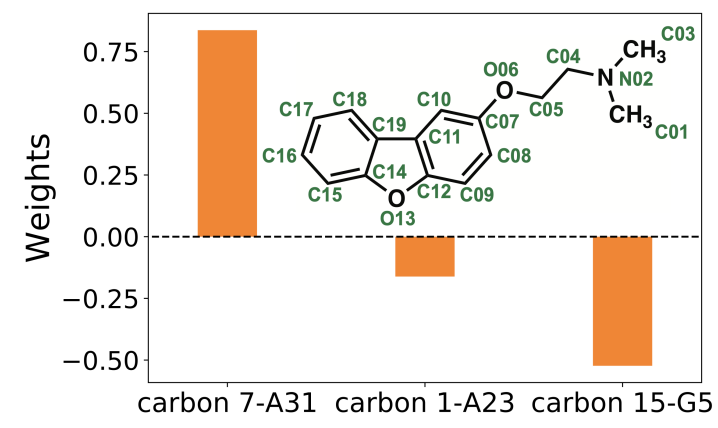

(b)

FIG. 3: Weights for different order parameters (OPs) learned by RAVE in the corresponding RC for riboswitch aptamer with (a) cognate ligand and (b) synthetic ligand. The OPs are named in the format "residue name-ligand atom". Ligand structures and atom names are shown in insets.

ber gradually decreases to 0 irrespective of which path is adopted during dissociation. We define the coordination number $C$ as:

$$
C=\frac{1}{N} \sum_{i=1}^{N} \sum_{j=1}^{M} \frac{1-\left(\frac{\mathbf{r}_{i j}}{r_{0}}\right)^{4}}{1-\left(\frac{\mathbf{r}_{i j}}{r_{0}}\right)^{8}}
$$

where $\mathrm{N}$ and $\mathrm{M}$ are the number of ligand and riboswitch atoms respectively. $r_{i j}$ is the distance between ligand atom $i$ and riboswitch atom $j$ and $r_{0}$ is set to be $3 \AA$. It can be seen from Fig. 4 that when bound to the riboswitch, the cognate ligand has higher $C$ relative to when the synthetic ligand is bound to it, illustrating that the synthetic ligand makes overall fewer contacts with the riboswitch, as also observed in crystal structures (PDB $6 \mathrm{E} 1 \mathrm{~W}$ and $6 \mathrm{E} 1 \mathrm{U})$. We can also see that the binding free energy difference between bound and unbound pose is $2 \sim 4 \mathrm{kcal} / \mathrm{mol}$ higher for the cognate ligand than that for the synthetic ligand. This is in quantitative agreement with relative affinity measurements $(3.0 \pm 1.4 \mathrm{kcal} / \mathrm{mol})$ through fluorescence titrations as reported in Ref. 25 .

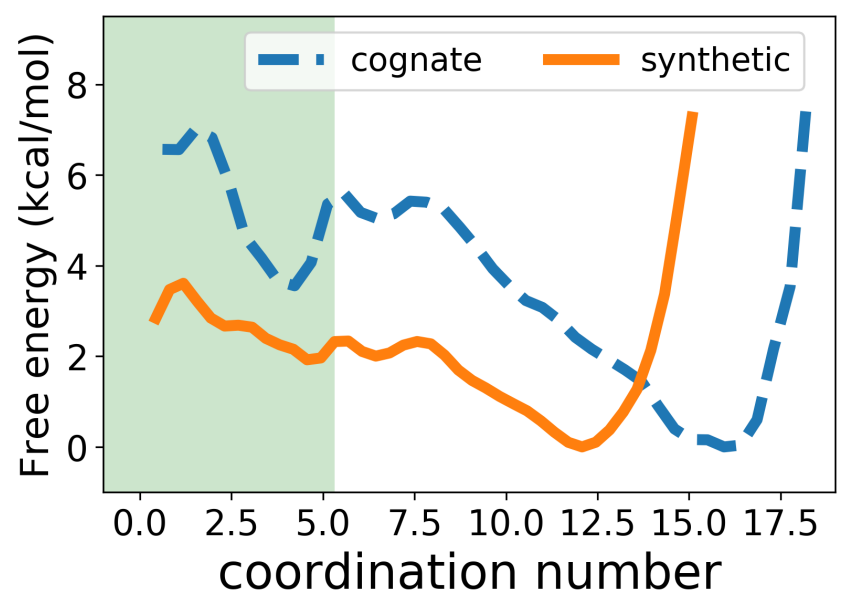

FIG. 4: Free energy profile of riboswitch with different ligands. Coordination number 0 (see Eq. 1) indicates ligand fully dissociated from riboswitch. The approximate range of coordination number corresponding to the unbound pose is highlighted in light green color $(C \lesssim 5.6)$.

\section{Predicting critical nucleotides for mutagenesis experiments}

We now analyze our 10 independent dissociation trajectories each obtained for the synthetic ligand bound and cognate ligand bound systems to predict critical nucleotides in the riboswitch that play a role in the dissociation process. We are interested in not just nucleotides we could have predicted from looking at the ligand-bound complexes, but also in nucleotides distal from the binding site. Such critical nucleotides are hard to predict from a purely structural perspective, but our all-atom resolution dissociation trajectories are ideally poised to make such predictions. Predictions made in this section are then validated through mutation experiments reported in Sec. IID.

From our collection of dissociation trajectories, we observe that dissociation mainly happens through two pathways (Fig. 5) and that for the cognate ligand, the top/blue pathway dominates, while for the synthetic ligand, the bottom/red pathway dominates. To better quantify the role played by different nucleotides, we monitor the change in the RMSD of every nucleotide during the dissociation process, relative to how the respective nucleotide is in the bound complex. Our motivation here is that the nucleotides showing greatest relative movement during the dissociation process are the ones most likely to be impacted once mutated. As shown in Supplementary Fig. 1, every nucleotide's RMSD changes with the coordination number $C$ which captures the extent of dissociation. These changes indicate different conformations the riboswitch adopts during ligand dissociation. Through this procedure we identify U22 and A32, highlighted in Fig. 2c, to be two such critical nucleotides for 


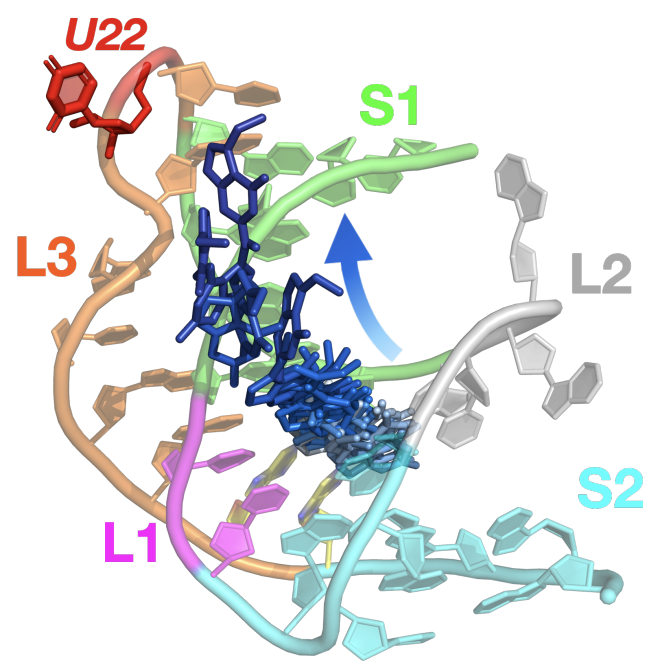

(a)

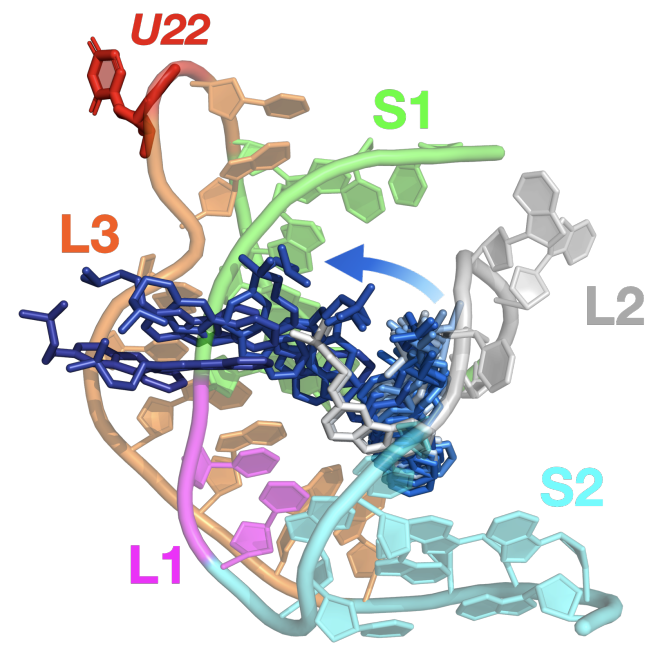

(c)

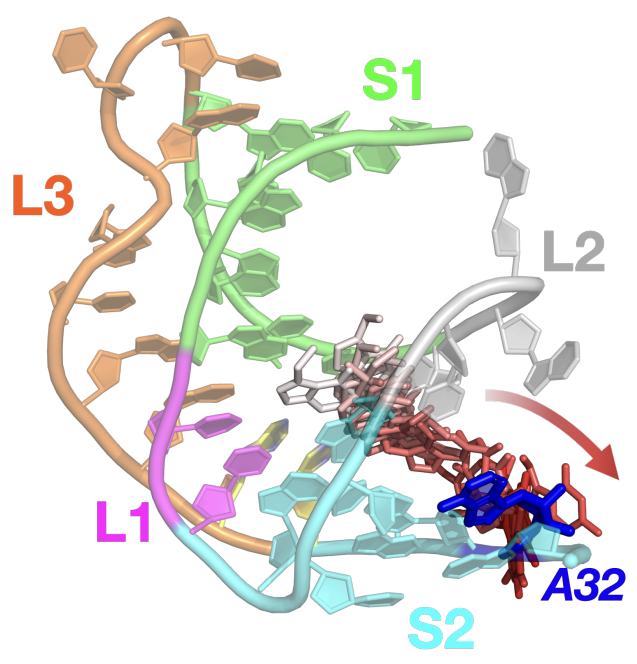

(b)

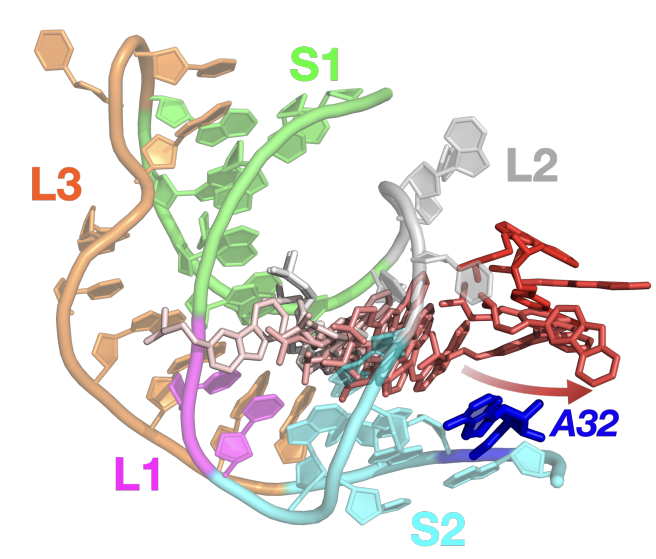

(d)

FIG. 5: Dissociation pathways for (a)-(b) cognate ligand and (c)-(d) synthetic ligand. Stem 1 (S1), stem 2 (S2), loop 1 (L1), loop2 (L2), and loop (L3) are colored green, cyan, magenta, gray, and orange respectively. During the dissociation processes, the ligands at different time frames are colored from white to dark blue and white to red, for the top pathway and bottom pathway respectively. The dissociation pathways are indicated with arrows. The predicted mutation sites U22 and A32 are highlighted in red and blue respectively.

the cognate and synthetic ligands respectively (see SM for further details of this protocol). Fig. 6 shows the changes in the RMSDs of these two selected nucleotides during the dissociation process. For U22, in RAVE simulations the RMSD increases much more for the cognate ligand-bound system, than for the synthetic ligandbound system. However, the reverse is found to be true for the nucleotide A32, which displays more enhanced movement during the dissociation of the synthetic ligand bound complex relative to the cognate ligand bound complex.

On the basis of the above observations, we can predict that for the cognate ligand, mutating U22 should display more tangible effects on the strength of the complex than mutating A32. For the synthetic ligand, we predict an opposite trend - mutating A32 should have a more pronounced effect on the interaction relative to mutating $\mathrm{U} 22$.

\section{Validating predicted critical nucleotides through mutagenesis experiments}

In Sec. II C we predicted on the behalf of our dissociation trajectories that mutating nucleotides U22 and A32 will have differing effects on the cognate ligandbound and synthetic ligand-bound systems. Here we report in vitro tests of our predictions. We measure 

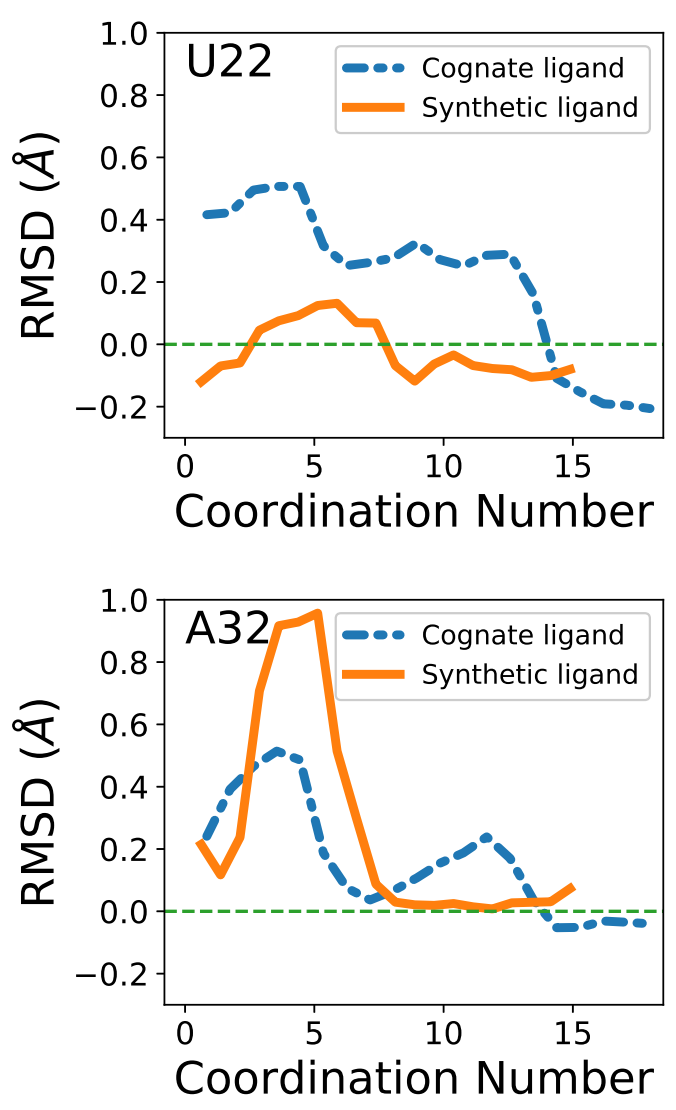

FIG. 6: Critical nucleotides prediction. Change in RMSD for nucleotides U22 and A32 during the dissociation process. RMSD profiles as function of coordination number are shown by blue dashed line and orange solid line for systems with cognate ligand and synthetic ligand separately.

the equilibrium dissociation constant $K_{D}$ for six different complexes, namely the two wild-type complexes, U22Acognate ligand, U22A-synthetic ligand, A32U-cognate ligand, and $\mathrm{A} 32 \mathrm{U}$-synthetic ligand. $K_{D}$ is the ratio between association rate $\left(k_{o n}\right)$ and dissociation rate $\left(k_{o f f}\right)$. In the low concentration limit, we do not expect that the presence of mutations will affect $k_{o n}$, which will be primarily diffusion limited, and attributable more to changes in the ligand than to mutations in the riboswitch. Thus our predicted mutations learned from dissociation trajectories should directly affect $k_{\text {off }}$ and hence $K_{D}$. By measuring the change of $K_{D}$, we can verify our predictions about how the mutations change the ligand dissociation process.

To experimentally test whether the A32U and U22A mutations impacted $K_{D}$ values, we used microscale thermophoresis (MST). $K_{D}$ values were measured using fluorescently labeled wild type, A32U, and U22A PreQ 1 riboswitch aptamer constructs for the cognate ligand. For $\mathrm{PreQ}_{1}$, we observed a $K_{D}$ of $11.7 \pm 0.35 \mathrm{nM}$ for the WT construct. In contrast, the $\mathrm{A} 32 \mathrm{U}$ mutant had a $K_{D}$ of

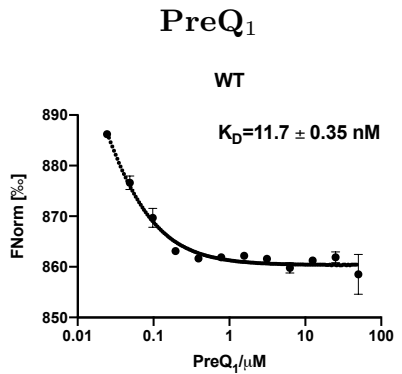

(a)

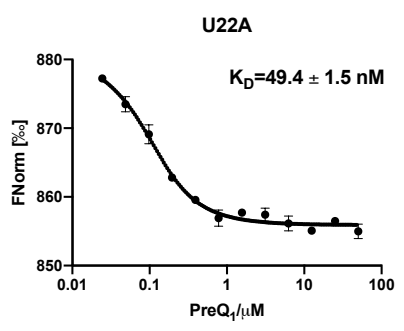

(b)

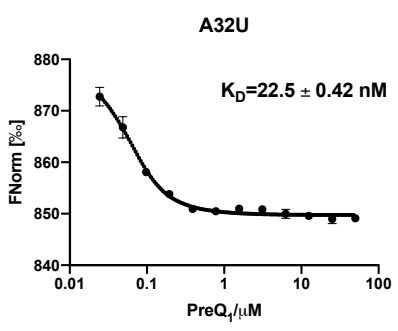

(c)
Dibenzofuran

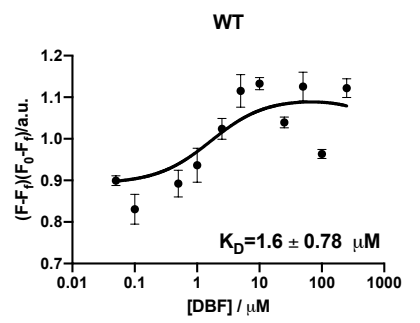

(d)

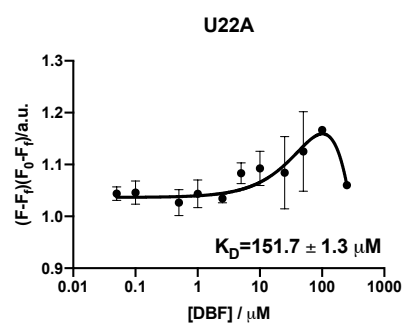

(e)

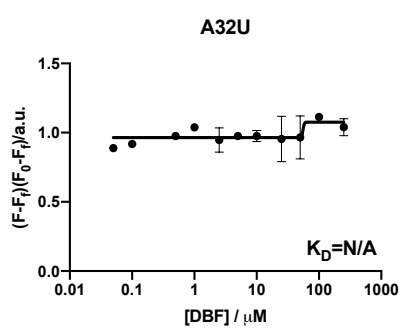

(f)
FIG. 7: Affinity measurement of $\mathrm{PreQ}_{1}$ (cognate) and dibenzofuran (synthetic) ligands binding to WT Tte riboswitch and U22A and A32U mutants. MST of 5'-cy5-labelled (a) WT Tte, (b) U22A, and (c) A32U mutants in the presence of PreQ 1 ligand. FIA of 5'-cy5-labelled (d) WT Tte, (e) U22A, and (f) A32U mutants in the presence of dibenzofuran. Error bars represent the standard deviation of three replicate experiments.

$22.5 \pm 0.42 \mathrm{nM}$ while the U22A mutant had a $K_{D}$ of $49.4 \pm 1.5 \mathrm{nM}$. For the synthetic ligand, changes were observed in fluorescence of the labeled RNA upon incubation with the ligand, and thus a fluorescence intensity assay (FIA) was used to measure $K_{D}$ values rather than MST. Using FIA we observed a $K_{D}$ of $1.6 \pm 0.7 \mu \mathrm{M}$ for the wild type aptamer. In contrast, both the U22A and A32U mutant constructs displayed significantly weaker $K_{D}$ values $(150 \pm 1.3 \mu \mathrm{M}$, and $\geq 100 \mu \mathrm{M}$ respectively). These $K_{D}$ measurements directly validate the findings from Sec. IIC. There we had predicted that mutating U22 will effect the cognate ligand-bound system more than mutating A32, and indeed we find that $K_{D}$ for U22A is 5 times weaker than WT, while only 2 times weaker for A32U. At the same time, we had predicted that mutating A32 will effect the synthetic ligand-bound system 
more than mutating U22, and indeed we find that while the $K_{D}$ for $\mathrm{U} 22 \mathrm{~A}$ is 75 times weaker than WT, there is effectively no tangible association at all for A32U with the synthetic ligand.

\section{CONCLUSION}

Over recent years a consensus view is emerging that a superior understanding of biomolecular structural dynamics, and not just static structures, can be fruitful in predicting key emergent biochemical and biophysical properties. ${ }^{32-34}$ However, in spite of the importance of the problem, tools to study biomolecular structural dynamics are still limited in their scope. This is true for proteins but even more so for RNAs, where it is especially challenging to determine the ensemble of possible structures that the RNA can adopt, and pinpoint the specific importance of different structures. ${ }^{33}$ In this work we demonstrate that this is indeed feasible - and how one can obtain detailed and robust insights into RNA structural dynamics through AI-augmented molecular simulations that maintain all-atom resolution for RNA, ions and all water molecules. All our predictions are validated through a gamut of complementary and experimental techniques that measure RNA flexibility and ligand binding. We specifically focused on the $T t$-PreQ $\mathrm{P}_{1}$ riboswitch aptamer system in its apo state and bound to two different ligands, where we first showed that $2 \mu s$ long unbiased MD simulations with classical all-atom force-fields for RNA and water can provide the same flexibility profile as measured in SHAPE experiments. Since the timescales for ligand dissociation are far slower than MD can typically access, we used the RAVE simulation method ${ }^{18}$ that enhances sampling along a low-dimensional reaction coordinate learned on-the-fly through the past-future information bottleneck framework. Through RAVE we obtain multiple independent dissociation trajectories for both cognate and synthetic liganded systems, demonstrating how the cognate and synthetic ligands invoke different aspects of the riboswitch's flexibility in order to dissociate. On this basis, we were able to predict pairs of mutations which would be expected to show contrasting behaviors for the cognate and synthetic ligands. The ability to make such predictions accurately and efficiently is of vital importance, as mutations that can disrupt RNA structural dynamics and ligand recognition processes have been linked to numerous human diseases. ${ }^{33,35}$ Our predictions for the $T t$-PreQ $Q_{1}$ aptamer were validated by designing mutant aptamers and performing affinity measurements.

Our work thus demonstrates a pathway to gain predictive insights, as opposed to retrospective validation, from enhanced molecular dynamics RAVE simulations that maintain femtosecond and all-atom resolution while directly observing processes as slow as ligand dissociation. While the focus in this work was on predicting the thermodynamically averaged aspects of riboswitch flexibility, in future work it should also be possible to directly calculate rate constants using complementary approaches and for more complicated RNA systems. More broadly, this work indicates that long time scale MD simulations can accurately model ligand dissociation from RNA, and provide validatable hypotheses about structural features that impact small molecule recognition of RNA. Thus, this strategy will be widely applicable not only to study natural systems such as riboswitches, but to more completely understand the structural features and dynamics that govern how synthetic small molecules interact with RNA.

\section{Supplementary material}

See supplementary material for biased MD trajectories, details of neural network architecture and other numerical details.

\section{Acknowledgements}

The authors thank Deepthought2, MARCC, and XSEDE (projects CHE180007P and CHE180027P) for providing computational resources used in this work. Y.W. would like to thank NCI-UMD Partnership for Integrative Cancer Research for financial support. This work was supported by the intramural program of the National Institutes of Health, National Cancer Institute, Center for Cancer Research (1 ZIA BC011585 07) (J.S.S. and S.P.) Research reported in this publication was supported by the National Institute Of General Medical Sciences of the National Institutes of Health under Award Number R35GM142719 (P.T.) The content is solely the responsibility of the authors and does not necessarily represent the official views of the National Institutes of Health. The authors thank Philip Homan for help analyzing SHAPE-MaP data and Alex Wilson for help setting up the simulations.

\section{Data availability statement}

The data that support the findings of this study are available from the corresponding author upon reasonable request.

\section{References}

${ }^{1}$ Nature Cell Biology 21, 535 (2019).

${ }^{2}$ N. S. De Groot, A. Armaos, R. Graña-Montes, M. Alriquet, G. Calloni, R. M. Vabulas, and G. G. Tartaglia, Nature communications 10, 1 (2019)

${ }^{3}$ S. M. Meyer, C. C. Williams, Y. Akahori, T. Tanaka, H. Aikawa, Y. Tong, J. L. Childs-Disney, and M. D. Disney, Chemical Society Reviews 49, 7167 (2020).

${ }^{4}$ J. P. Falese, A. Donlic, and A. E. Hargrove, Chemical Society Reviews 50, 2224 (2021).

${ }^{5}$ B. J. Tucker and R. R. Breaker, Current opinion in structural biology 15, 342 (2005).

${ }^{6}$ E. Lekka and J. Hall, FEBS letters 592, 2884 (2018).

${ }^{7}$ M. Esteller, Nature reviews genetics 12, 861 (2011).

${ }^{8}$ K. F. Blount and R. R. Breaker, Nature biotechnology 24, 1558 (2006).

${ }^{9}$ C. M. Connelly, M. H. Moon, and J. S. Schneekloth Jr, Cell chemical biology 23, 1077 (2016). 
${ }^{10}$ A. U. Juru and A. E. Hargrove, Journal of Biological Chemistry 100191 (2021).

${ }^{11}$ N. G. Walter, Methods 49, 85 (2009), rNA Dynamics.

${ }^{12}$ K. M. Weeks, Current opinion in structural biology 20, 295 (2010).

${ }^{13}$ A. Serganov and E. Nudler, Cell 152, 17 (2013).

${ }^{14}$ C. M. Connelly, T. Numata, R. E. Boer, M. H. Moon, R. S. Sinniah, J. J. Barchi, A. R. Ferré-D'Amaré, and J. S. Schneekloth, Nature Communications 10, 1501 (2019).

${ }^{15} \mathrm{~A}$. Roth and R. R. Breaker, Annual review of biochemistry 78, 305 (2009).

${ }^{16}$ K. C. Suddala, A. J. Rinaldi, J. Feng, A. M. Mustoe, C. D. Eichhorn, J. A. Liberman, J. E. Wedekind, H. M. Al-Hashimi, C. L. Brooks III, and N. G. Walter, Nucleic acids research $4 \mathbf{1}$, 10462 (2013).

${ }^{17}$ G. M. Schroeder, D. Dutta, C. E. Cavender, J. Jenkins, E. M. Pritchett, C. D. Baker, J. M. Ashton, D. H. Mathews, and J. E. Wedekind, Nucleic Acids Research 48, 8146 (2020), https://academic.oup.com/nar/articlepdf/48/14/8146/34131194/gkaa546.pdf.

${ }^{18}$ Y. Wang, J. M. L. Ribeiro, and P. Tiwary, Nat. Commun. 10, 3573 (2019).

${ }^{19}$ P. Ravindra, Z. Smith, and P. Tiwary, Mol. Syst. Des. Eng. 5, 339 (2020).

${ }^{20}$ A. Barducci, G. Bussi, and M. Parrinello, Phys. Rev. Lett. 100, 020603 (2008).

${ }^{21}$ O. Valsson, P. Tiwary, and M. Parrinello, Annual Review of Physical Chemistry 67, 159 (2016), pMID: 26980304, https://doi.org/10.1146/annurev-physchem-040215-112229.

${ }^{22}$ N. A. Siegfried, S. Busan, G. M. Rice, J. A. E. Nelson, and K. M.
Weeks, Nature Methods 11, 959 (2014).

${ }^{23}$ M. J. Smola, G. M. Rice, S. Busan, N. A. Siegfried, and K. M. Weeks, Nature Protocols 10, 1643 (2015).

${ }^{24}$ M. H. Moon, T. A. Hilimire, A. M. Sanders, and J. S. Schneekloth Jr, Biochemistry 57, 4638 (2018).

${ }^{25}$ C. M. Connelly, T. Numata, R. E. Boer, M. H. Moon, R. S. Sinniah, J. J. Barchi, A. R. Ferré-D'Amaré, and J. S. Schneekloth, Nature Communications 10, 1501 (2019).

${ }^{26}$ V. Mlỳnskỳ and G. Bussi, Current opinion in structural biology 49, 63 (2018).

${ }^{27}$ D. Wang and P. Tiwary, The Journal of Chemical Physics 154, 134111 (2021).

${ }^{28}$ T. Marinus, A. B. Fessler, C. A. Ogle, and D. Incarnato, Nucleic acids research 49, e34 (2021).

${ }^{29}$ G. M. Schroeder, D. Dutta, C. E. Cavender, J. L. Jenkins, E. M. Pritchett, C. D. Baker, J. M. Ashton, D. H. Mathews, and J. E. Wedekind, Nucleic acids research 48, 8146 (2020).

${ }^{30}$ G. Pinamonti, S. Bottaro, C. Micheletti, and G. Bussi, Nucleic acids research 43, 7260 (2015).

${ }^{31}$ D. Tan, S. Piana, R. M. Dirks, and D. E. Shaw, Proceedings of the National Academy of Sciences 115, E1346 (2018), https://www.pnas.org/content/115/7/E1346.full.pdf.

${ }^{32}$ A. M. Mustoe, C. L. Brooks, and H. M. Al-Hashimi, Annual Review of Biochemistry 83, 441 (2014), pMID: 24606137, https://doi.org/10.1146/annurev-biochem-060713-035524.

${ }^{33}$ L. R. Ganser, M. L. Kelly, D. Herschlag, and H. M. Al-Hashimi, Nature reviews Molecular cell biology 20, 474 (2019).

${ }^{34}$ M. Gruebele, "Protein dynamics in simulation and experiment," (2014).

${ }^{35}$ M. Halvorsen, J. S. Martin, S. Broadaway, and A. Laederach, PLoS genetics 6, e1001074 (2010). 